\title{
Aberrant activity and connectivity of the posterior superior temporal sulcus during social cognition in schizophrenia
}

\author{
Daniela Mier ${ }^{1} \cdot$ Sarah Eisenacher $^{2} \cdot$ Franziska Rausch $^{2} \cdot$ Susanne Englisch $^{2}$. \\ Martin Fungisai Gerchen ${ }^{1} \cdot$ Vera Zamoscik $^{1} \cdot$ Andreas Meyer-Lindenberg $^{2}$ • \\ Mathias Zink ${ }^{2} \cdot$ Peter Kirsch ${ }^{1}$
}

\begin{abstract}
Schizophrenia is associated with significant impairments in social cognition. These impairments have been shown to go along with altered activation of the posterior superior temporal sulcus (pSTS). However, studies that investigate connectivity of pSTS during social cognition in schizophrenia are sparse. Twenty-two patients with schizophrenia and 22 matched healthy controls completed a social-cognitive task for functional magnetic resonance imaging that allows the investigation of affective Theory of Mind (ToM), emotion recognition and the processing of neutral facial expressions. Moreover, a resting-state measurement was taken. Patients with schizophrenia performed worse in the social-cognitive task (main effect of group). In addition, a group by social-cognitive processing interaction was revealed for activity, as well as for connectivity during the social-cognitive task, i.e., patients with schizophrenia showed hyperactivity of right pSTS during neutral face processing, but hypoactivity during emotion recognition and affective ToM. In addition, hypoconnectivity between right and left pSTS was revealed for affective ToM, but
\end{abstract}

Mathias Zink and Peter Kirsch have contributed equally to this work.

Daniela Mier

Daniela.Mier@zi-mannheim.de

Department of Clinical Psychology, Central Institute of Mental Health, University of Heidelberg/Medical Faculty Mannheim, J5, 68159 Mannheim, Germany

2 Department of Psychiatry and Psychotherapy, Central Institute of Mental Health, University of Heidelberg/Medical Faculty Mannheim, J5, 68159 Mannheim, Germany not for neutral face processing or emotion recognition. No group differences in connectivity from right to left pSTS occurred during resting state. This pattern of aberrant activity and connectivity of the right pSTS during social cognition might form the basis of false-positive perceptions of emotions and intentions and could contribute to the emergence and sustainment of delusions.

Keywords Schizophrenia $\cdot$ Social cognition $\cdot$ Posterior superior temporal sulcus · Functional magnetic resonance imaging · Psychophysiological interactions · Resting-state connectivity

\section{Introduction}

Schizophrenia is a severe psychiatric disorder associated with significant deficits in social functioning that have been related to impaired social-cognitive functions [1, 2]. While several studies investigated the neural bases of altered social cognition in schizophrenia in terms of activity, little is known about the connectivity of core social-cognitive regions. In this context, a brain region that is currently getting into the focus is the posterior superior temporal sulcus (pSTS). Until now, however, it is unclear whether deficient functioning of the pSTS presents a global marker of schizophrenia, or is specific for the social-cognitive deficits.

Deficits in social cognition (the processing of information that leads to the recognition of emotions and intentions of others; [3]) have been consistently reported in schizophrenia. These deficits range from alterations in face processing $[4,5]$, over emotion recognition [6], up to deficits in complex social cognitions [7], such as in Theory of Mind (ToM; the ability to recognize mental states, such as wishes, desires and intentions; [8]). Importantly, 
patients with schizophrenia are impaired in both, cognitive (the ability to understand mental states without relying on emotional information) and affective (the ability to recognize affective mental states) ToM, while the group around Shamay-Tsoory [9] even showed pronounced deficits in affective ToM in schizophrenia. Since deficits in social cognition can even be found in remitted schizophrenia patients [10], as well as in people with an at-riskmental state for schizophrenia [11], and in relatives of schizophrenia patients [12], social-cognitive impairments can be assumed to present an intermediate phenotype [13, 14] and trait marker of the disease [15]. In agreement with this assumption, several studies found evidence for alterations in the neural processing of social stimuli in schizophrenia.

Meta-analyses suggest alterations in amygdala activation in schizophrenia during face processing and emotion recognition [16]. Interestingly, current studies [17, 18] as well as a recent meta-analysis [19] revealed relative hyperactivity of the amygdala in response to neutral facial expressions in schizophrenia and a relative hypoactivity in response to emotional facial expressions. Hence, the amygdala might be one of the major candidates to explain deficient social cognition, as well as deficient social functioning in schizophrenia [20]. The pSTS, another core area for social cognition [21], presents a comparable pattern of activation differences in schizophrenia: Again, there is evidence for hyperactivity in response to neutral facial expressions, respectively, in non-explicitly emotional control conditions of social-cognitive tasks and relative hypoactivity in response to emotional facial expressions [4, 22, 23]. For the amygdala, activity and connectivity during emotional face processing in schizophrenia were shown to be dependent of the state of disease and could not be revealed for relatives of schizophrenia patients [24]. In contrast, there is some evidence for a state independence with regard to pSTS activation, because also carriers of schizophrenia risk alleles show altered STS activation during social-cognitive tasks [25]. Thus, aberrations in STS activation might constitute the intermediate phenotype of social-cognitive deficits of schizophrenia and could contribute to the emergence of different schizophrenia symptoms [26].

The pSTS, however, is not only involved in social cognition, but also in the processing of biological movement, has a central functioning in attentional control and serves as a brain region for multisensory integration [21]. In consequence, social-cognitive deficits in schizophrenia might not necessarily constitute an inherent deficit of social cognition alone, but also deficits in attentional or cognitive processes might negatively influence social-cognitive processes via aberrant pSTS functioning. In this case, not only the activity of the pSTS would be important, but also its connectivity to brain regions of the social-cognitive, as well as other networks, such as the executive control network [27, 28].

Altered STS-frontal connectivity can be detected in subjects with at-risk mental states for schizophrenia already [29]. In addition, in some of the few studies that investigated connectivity during social-cognitive tasks in schizophrenia, aberrant pSTS [30, 31] as well as aberrant amygdala connectivity was found [32, 33]. Interestingly, in both studies that revealed aberrant pSTS connectivity, the hyperconnectivity of the pSTS only occurred while patients solved the control condition of the social-cognitive paradigm. This finding has at least two possible explanations: First, it might be that high pSTS connectivity presents the default state in schizophrenia, but does not increase with task demands. In this case, it would only be detectable in tasks without a social-cognitive (or a general cognitive) demand and is not revealed in tasks that require an active engagement of the social brain network (i.e., superior temporal sulcus, amygdala, medial prefrontal cortex, insula, inferior prefrontal cortex/premotor cortex [3, 34, 35]) in healthy participants, too. Second, the pSTS hyperactivity as well as hyperconnectivity reflects the schizophrenia brain's basis for hypermentalizing. Frith and Corcoran [36] as well as others assumed that patients with paranoid schizophrenia tend to hypermentalize. Since the pSTS is constantly found to be of relevance in studies investigating intention recognition $[37,38]$, hyperengagement of the pSTS in schizophrenia might lead to false-positive perceptions of intentions, i.e., to hypermentalizing.

In summary, people with schizophrenia show profound deficits in social cognition. These deficits are reflected in altered amygdala and pSTS activation as well as altered connectivity of these areas. What is not clear, however, is whether the altered pSTS activity and connectivity present a default state of the schizophrenia brain, or might specifically reflect the neural basis for hypermentalizing. To address this question, patients with schizophrenia and a healthy control group underwent resting-state functional magnetic resonance imaging (fMRI), as well as a socialcognitive paradigm. The applied social-cognitive paradigm allows the investigation of affective ToM, emotion recognition and neutral face processing, and has already revealed hyperactivity of amygdala and pSTS during the processing of neutral facial expression and emotion recognition, but not during affective ToM in schizophrenia outpatients [4]. The aim of the present study was replicating these findings in an independent sample of patients with schizophrenia and healthy controls. In addition, the study design allowed us to address the following additional questions: If we replicate the findings of Mier et al. [4], does the aberrant activation go along with altered connectivity during social-cognitive 
processing in schizophrenia? Do we find comparable or different aberrations in connectivity during rest?

\section{Methods and materials}

\section{Sample}

The present study is part of a larger investigation of metacognitive deficits in schizophrenia (for details see [39, 40]) and was approved by the local ethics board of the Medical Faculty Mannheim, University of Heidelberg (AZ 2009296N-MA). Twenty-two patients with schizophrenia and 22 healthy control subjects participated in the study. Patients with schizophrenia were recruited via the wards of the Central Institute of Mental Health, Mannheim, Germany. Patients with schizophrenia were included when having a diagnosis of schizophrenia according to the Diagnostical and Statistical Manual of Mental Disorders-IV (DSM-IV; [41]) which was confirmed by the attending physician and an experienced clinical rater (psychologists as well as psychiatrists with formal training). A number of patients were diagnosed with comorbid disorders. Psychiatric comorbidities included obsessive compulsive disorder $(n=1)$, pathological gambling $(n=1)$, adjustment disorder $(n=1)$, and depression $(n=1)$. Five patients were diagnosed with substance abuse disorders (multiple substances: $n=2$, analgesics: $n=1$, alcohol: $n=1$, cannabis: $n=1$ ). Two patients had a history of substance abuse and one patient of benzodiazepine dependence, but these patients did not fulfill this diagnosis at present. All patients were abstinent at the time of testing. In addition, patients were interviewed by the experienced clinical raters with the Positive and Negative Syndrome Scale (PANSS [42]) and the Scale for the Assessment of Negative Symptoms (SANS [43]) to assess current positive and negative, as well as general psychopathology. Social functioning was assessed with the Personal and Social Performance Scale (PSP [44]). All patients received antipsychotic medication with no changes in substances and no changes in dosage larger than $25 \%$ within the last 2 weeks. Healthy controls were recruited via announcements in local newspapers, the homepage of the Central Institute of Mental Health, as well as via flyers. Healthy controls were screened with the Mini International Neuropsychiatric Interview (MINI [45]) to exclude past or present psychiatric disorders. Moreover, healthy controls were only included when reporting not having a first degree relative with schizophrenia or bipolar disorder. Healthy controls were matched to the schizophrenia patients by age, gender, education and estimated verbal intelligence (as assessed with the Multiple-word-choice-test, Mehrfachwahl-Wortschatz-Test; MWTB [46]). Exclusion criteria for both groups were neurological diseases, current (for the healthy control group also past) substance abuse or dependence except for nicotine, as well as contraindications for magnetic resonance imaging. All participants gave written informed consent before being enrolled in the study. Characteristics of both groups, medication, as well as clinical ratings are reported in Table 1.

\section{Social-cognitive testing}

Participants were tested with the Meyer-Salovey-Caruso emotional intelligence test (MSCEIT) to assess social and emotional managing skills [47, 48]. The MSCEIT is a widely used test to investigate social cognition in schizophrenia (e.g., [47]). The subtest managing emotions that was used in the present study can be divided into two tasks, namely emotion management (regulation of one's own emotions) and emotional relations (managing emotions to achieve an outcome involving other people). Eight scenarios are read out loud to the participant, each followed by three to four possible reactions of the protagonist. Each reaction has to be evaluated according to its effectiveness of solving the situation by regulating emotions. The individual scores are weighed according to normative data to reach a total $t$ value. Additional assessments of social cognition were completed with a computerized version of the reading the mind in the eyes test (RMET; [49]) to investigate ToM, as well as with the e-scale to investigate empathy [50]. In the RMET, excerpts of eyes (equal number of male and female) are shown to participants on a computer screen. Beneath, four adverbs are presented. Participants are asked to decide by a click on the word which of the adverbs best described what the person on the picture (by means of his/her eyes) thinks, feels or expresses. The total number of correct and incorrect decisions is calculated. In sum, 37 pictures must be evaluated, of which the first one is a practice trial which is not evaluated. Using this version of the RMET, several studies have already revealed significant differences between patients with schizophrenia and healthy controls $[51,52]$. The e-scale is a questionnaire to assess empathy, consisting of 25 items. On 5-point scales, participants are asked to rate in how far the statements apply to them personally $(0=$ not applicable to $5=$ totally applicable). A sumscore is calculated. While the different factors of the e-scale could not be reliably replicated, the e-scale seems to represent a general empathy factor [50].

\section{Experimental paradigm}

The experimental paradigm for fMRI allows the investigation of three functions of social cognition: face processing, emotion recognition and affective ToM [4, 53]. For this purpose, participants are presented with statements that are immediately followed by facial expressions [38]. These statements refer to either a physical feature (gender, 
Table 1 Characteristics of both groups, as well as clinical data for the patient group, reported in means and standard deviations (SD) in brackets

\begin{tabular}{|c|c|c|c|}
\hline & Schizophrenia patients & Healthy controls & Statistics \\
\hline Gender & $18 \mathrm{f}, 4 \mathrm{~m}$ & $16 \mathrm{f}, 6 \mathrm{~m}$ & Chi, $p=0.72$, n.s. \\
\hline Age (years) & $38.05(9.19)$ & $37.50(10.82)$ & $p=0.86, n . s$. \\
\hline School education (years) & $10.36(1.65)$ & $10.95(1.57)$ & $p=1.00, n . s$. \\
\hline Verbal intelligence (IQ) & $97.23(10.83)$ & $101.68(10.06)$ & $p=0.17$, n.s. \\
\hline Age of onset & $28.0(8.80)$ & & \\
\hline Duration of illness (years) & $10.50(6.84)$ & & \\
\hline \multirow[t]{8}{*}{ Antipsychotic medication } & Amisulpride: $n=2$ & & \\
\hline & Aripiprazole: $n=2$ & & \\
\hline & Clozapine: $n=5$ & & \\
\hline & Olanzapine: $n=2$ & & \\
\hline & Paliperidone: $n=1$ & & \\
\hline & Quetiapine: $n=4$ & & \\
\hline & Risperidone: $n=5$ & & \\
\hline & Study medication: $n=1$ & & \\
\hline Benzodiazepines & Lorazepam: $n=7$ & & \\
\hline Duration of antipsychotic dosage & Median: 6 days & & \\
\hline CPZ equivalent ( $\mu \mathrm{g} / \mathrm{ml})$ & $452.52(176.02)$ & & \\
\hline PANSS global & $32.59(7.14)$ & & \\
\hline PANSS positive & $14.32(3.87)$ & & \\
\hline SANS & $23.05(19.08)$ & & \\
\hline PSP & $54.50(12.47)$ & & \\
\hline
\end{tabular}

PANSS Positive and Negative Syndrome Scale, SANS Scale for the Assessment of Negative Symptoms, $P S P$ Personal and Social Performance Scale, $C P Z$ chlorpromazine hair color, age) of the person whose facial expression is displayed (face processing), an emotional state (fear, happiness, anger) (emotion recognition), or an intention (running away, cheering, blustering) of the displayed person (affective ToM). The type of facial expression depends on the task condition: A neutral facial stimulus is presented for face processing, whereas an emotional one is displayed for emotion recognition and affective ToM. Participants were instructed to indicate by button press whether the statement matches the displayed person. For each of the social-cognitive functions, half of the facial stimuli were female, half male. Further, in affective ToM and emotion recognition the emotions fear, anger and happiness were shown and were equally distributed within the conditions. In the neutral face processing condition, only neutral facial expressions were shown. Statements, as well as facial stimuli, were presented for $2 \mathrm{~s}$. Trials were separated by a fixation cross with a variable presentation time of $2 \mathrm{~s}$ on average (with a jitter of 1-3 s). A total of 30 trials were presented for each socialcognitive function, resulting in an experimental time of 9.5 min (experimental design is presented in Fig. 1). Analyses of emotion recognition and affective ToM were accomplished across emotions. The experiment was presented with the software Presentation (Neurobehavioral Systems, Albany, CA). Participants responded with a current design response device (Current Designs, Inc., Philadelphia, PA) and watched the experiment via VisuaStim video goggles (Resonance Technology Inc, Northridge, USA).

\section{Data acquisition}

FMRI data were acquired with a 3-Tesla Siemens Tim TRIO (Siemens, Erlangen). Prior to the experiment, a magnetization-prepared rapid gradient-echo (MPRAGE) anatomical scan was acquired to investigate potential gross brain abnormalities (slices: 192; repetition time (TR): $2300 \mathrm{~ms}$; voxel size: $1 \times 1 \times 1 \mathrm{~mm}$ ). For the experimental design, an echo planar imaging sequence was applied (slices: 32; TR: $2000 \mathrm{~ms}$; echo time (TE): $30 \mathrm{~ms}$; flip angle: $80^{\circ}$; voxel size: $3 \times 3 \times 3 \mathrm{~mm}$ (with 1-mm gap); matrix: $64 \times 64 \mathrm{~mm}$ ) with 275 scans (duration $9.2 \mathrm{~min}$ ). An additional echo planar image (EPI) was taken without any task to analyze resting-state connectivity (slices: 28 ; TR: $2000 \mathrm{~ms}$; TE: $30 \mathrm{~ms}$; flip angle: $80^{\circ}$; voxel size: $3 \times 3 \times 4$ (with 1-mm gap); matrix: $64 \times 64 \mathrm{~mm}$ ). Two hundred and four scans were collected for the resting state (duration $6.8 \mathrm{~min}$ ). During the resting-state measure, participants were lying still in the scanner with their eyes open, focusing on a fixation cross, while letting their mind wander. The resting-state measurement was taken after the experimental task. To allow physiological artifact correction of the resting-state data, heart rate and respiration rate were sampled 
Fig. 1 Experimental design exemplarily depicted for faces showing the emotion fear

\section{Statement} $2 \mathrm{sec}$

Emotion

ToM

Neutral
This person is female

\section{Picture $\max 2$ sec}
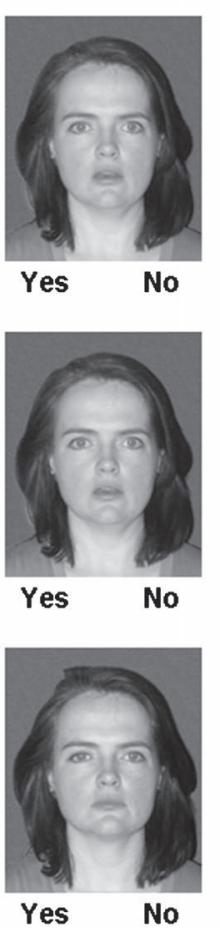

ITI

$0.5-3.5 \mathrm{sec}$
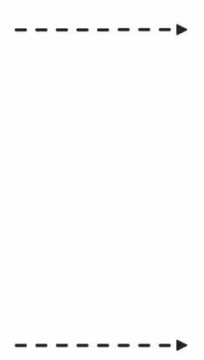

at $50 \mathrm{~Hz}$ with the scanner built-in equipment. For the EPIs, the first four scans were discarded to account for T1 saturation effects.

\section{Data analysis}

Functional magnetic resonance imaging data were analyzed with SPM8 (Wellcome Trust Centre for Neuroimaging, University College London, United Kingdom). Data were preprocessed with slice time correction, realignment, normalization and smoothing (9-mm isotropic kernel). Only participants with translation $<3 \mathrm{~mm}$ and rotation $<3^{\circ}$ were included for further analyses. Movement parameters did not differ significantly between groups (all $p \mathrm{~s}>.163$ ). First-level analyses to model brain activation in response to the experiment were applied with three regressors for the social-cognitive functions, as well as six regressors derived from realignment to account for possible residual movement-related variance. Data were filtered with a 128-s highpass filter. For the connectivity analyses, we used a datadriven approach, applying a functional mask of the right pSTS cluster that showed a significant group by social-cognitive function interaction (see below in the results section) as seed region. Connectivity of this right pSTS cluster was investigated with generalized psychophysiological interactions (gPPI [54]) ran in the gPPI toolbox (http://www.nitrc. org/projects/gppi). Here, we were interested in the group by social-cognitive function interaction, too, i.e., changes in connectivity during affective ToM in comparison with emotion recognition and neutral face processing, depending on the group status. To this end, the first eigenvariate of the cluster that showed a significant social-cognitive function by group interaction of the right pSTS activation was extracted for each person (eigenvariate extraction was achieved without applying a significance threshold), deconvolved by the canonical hemodynamic response function (HRF) and multiplied with the time series of affective ToM, emotion recognition and face processing, respectively, to represent condition-specific interactions. These interaction regressors were subsequently convolved again with the HRF. Next, a gPPI first-level analysis was set up, containing the interaction regressors, the task regressors and the (unmultiplied) first eigenvariate of pSTS activation.

Complete sets of resting-state data including the physiological measures were available for 13 patients with schizophrenia (3 females) and 19 healthy controls (5 females). For the analysis of the resting-state data, first physiological artifacts were corrected using the Aztec software tool [55], including a high-pass filter of 512 s. Preprocessing included realignment, slice time correction, normalization and smoothing with a 9-mm Gaussian kernel. For the functional connectivity analysis of the resting-state data, first eigenvariate was extracted from the time course of the same pSTS mask that was used for the gPPI-analysis, as 
Table 2 Performance in the social-cognitive tasks for both groups, reported in means and standard deviations (SD) in brackets

\begin{tabular}{|c|c|c|c|}
\hline & Schizophrenia patients & Healthy controls & Statistics \\
\hline Affective ToM (\%) & $64.70(9.79)$ & $71.81(7.95)$ & $p=0.011, d=.77$ \\
\hline Emotion recognition $(\%)$ & $66.06(9.85)$ & $73.79(8.38)$ & $p=0.008, d=.64$ \\
\hline Neutral (\%) & $57.72(11.01)$ & $60.76(7.42)$ & $p=0.289, n . s ., d=.33$. \\
\hline Affective ToM (RTs) & $1234.49(154.12)$ & $1174.80(175.31)$ & $p=0.237, n . s ., d=.36$ \\
\hline Emotion recognition (RTs) & $1264.19(184.29)$ & $1255.46(166.49)$ & $p=0.870, n . s ., d=.05$ \\
\hline Neutral (RTs) & $1105.87(162.84)$ & $1022.64(182.20)$ & $p=0.118, n . s ., d=.48$ \\
\hline MSCEIT emotion & $89.36(10.10)$ & $90.91(7.90)$ & $p=0.575, n . s ., d=.17$ \\
\hline MSCEIT social & $84.41(9.86)$ & $89.82(10.65)$ & $p=0.088, n . s ., d=.53$ \\
\hline RMET & $23.00(3.56)$ & $23.18(6.69)$ & $p=0.869, n . s ., d=.03$ \\
\hline Empathy & $78.64(14.85)$ & $81.59(14.41)$ & $p=0.507$, n.s.,$d=.20$ \\
\hline
\end{tabular}

Statistics were conducted with $t$ tests

Affective ToM affective Theory of Mind, Neutral neutral face processing, RTs reaction times, MSCEIT emotion emotional management scale of the Meyer-Salovey-Caruso emotional intelligence test, MSCEIT social social management scale of the Meyer-Salovey-Caruso emotional intelligence test, RMET reading the mind in the eyes test, Empathy e-scale well as from white matter and cerebrospinal fluid (CSF) masks. The individual pSTS, white matter and CSF first eigenvariates together with the six movement regressors were entered into first-level regression analyses in SPM8 to investigate the covariation between pSTS activity and activity in all other brain regions.

Second-level analysis consisted of the application of a flexible-factorial design with the factors subject, socialcognitive function and group for task-related activity and connectivity. Resting-state connectivity was compared between groups by $t$ tests. To achieve the balance between false-positive and false-negative findings, significance threshold for whole brain analyses was set to $p<0.005$, cluster size $(k)=20$ [56]. Post hoc tests for the analysis of the group by social-cognitive function interaction in right pSTS (activity) and in left pSTS (connectivity) were achieved by extracting the first eigenvariate of the interaction clusters for each social-cognitive function (affective ToM, emotion recognition and neutral face processing) for each participant and by subjecting the extracted eigenvariates to $t$ tests in SPSS (version 20). The eigenvariate of the left pSTS interaction cluster was also extracted for the resting-state measure to compare connectivity differences between task and rest (see supplementary text). No significance threshold was used for eigenvariate extraction.

Behavioral data were analyzed with SPSS, too. Performance data as well as reaction times were analyzed with repeated measures analyses of variance (ANOVAs) and according post hoc $t$ tests. RMET was analyzed between groups with a $t$ test. The same was true for the MSCEIT and the e-scale. Correlations between measures of socialcognitive performance, psychopathology, age of onset and duration of illness, as well as with chlorpromazine equivalents (i.e., potency of the antipsychotic medication [57]) were assessed with Pearson correlation coefficients. Significance threshold was set to $p<0.05$.

\section{Results}

\section{Social-cognitive tasks}

No significant differences between groups occurred for the RMET task, as well as for the e-scale. In the MSCEIT, patients were found to perform equally to healthy controls in the emotional managing scale, but performed trendwise worse in the social managing scale (see Table 2). For the social-cognitive fMRI task, a significant main effect of group occurred with the schizophrenia group, having a significantly lower performance than the healthy control group $\left[F(d f 42,1)=9.57, p=0.004, \eta^{2}=.19\right]$. The group $\times$ social-cognitive function interaction was not significant. Patients did not differ significantly from the control group in reaction times. Across all participants, the affective ToM performance was significantly correlated with the RMET performance $(r=.47, p<0.001)$ and the MSCEIT social managing scale $(r=.44, p=0.003)$, while the emotion recognition performance correlated only with the RMET performance $(r=.42, p=0.004)$. Analysis of the association of chlorpromazine equivalents and behavior showed no significant association with performance. There was a negative correlation of RMET performance and SANS $(r=-.43, p=0.044)$, and of the PANSS negative scale and emotional managing $(r=-.46, p=0.033)$. Further, there was a negative correlation of age of onsets and MSCEIT emotional managing $(r=-.45, p=0.035)$, 
Table 3 Functional brain imaging results for the effect of ToM $(p<0.005$ uncorrected, $k=20)$ and the group by condition interaction $(p<0.005$ uncorrected, $k=20$ )

\begin{tabular}{|c|c|c|c|c|c|c|c|}
\hline \multirow[t]{2}{*}{ Area } & \multirow[t]{2}{*}{ BA } & \multirow[t]{2}{*}{ Cluster } & \multicolumn{3}{|c|}{ MNI } & \multirow[t]{2}{*}{$t$ value } & \multirow[t]{2}{*}{$p$ value } \\
\hline & & & $x$ & $y$ & $z$ & & \\
\hline \multicolumn{8}{|l|}{ ToM $>$ Emo $>$ neutral } \\
\hline Inferior frontal gyrus & 45 & 311 & 56 & 32 & 6 & 7.29 & $p<0.001$ \\
\hline Inferior frontal gyrus & 47 & 506 & -48 & 30 & -4 & 5.72 & $p<0.001$ \\
\hline Inferior frontal gyrus & 45 & & -52 & 26 & 6 & 4.99 & $p<0.001$ \\
\hline Superior temporal gyrus & 22 & 781 & -58 & -54 & 6 & 5.34 & $p<0.001$ \\
\hline Middle temporal gyrus & 39 & & -40 & -60 & 18 & 3.05 & 0.002 \\
\hline Middle temporal gyrus & 21 & & -46 & -34 & -6 & 2.66 & 0.005 \\
\hline Middle temporal gyrus & 21 & 1.510 & 52 & -16 & -12 & 5.14 & $p<0.001$ \\
\hline Middle temporal gyrus & 22 & & 50 & -42 & 2 & 4.96 & $p<0.001$ \\
\hline Middle temporal gyrus & 21 & & 52 & -2 & -22 & 4.91 & $p<0.001$ \\
\hline Superior temporal gyrus & 38 & 452 & -48 & 14 & -30 & 4.98 & $p<0.001$ \\
\hline Middle temporal gyrus & 21 & & -54 & -4 & -20 & 4.93 & $p<0.001$ \\
\hline Middle temporal gyrus & 21 & & -52 & -12 & -16 & 4.73 & $p<0.001$ \\
\hline Fusiform gyrus & 37 & 102 & 44 & -50 & -18 & 4.50 & $p<0.001$ \\
\hline Fusiform gyrus & 37 & & 46 & -40 & -18 & 3.57 & $p<0.001$ \\
\hline Amygdala & & 63 & 20 & -8 & -16 & 4.11 & $p<0.001$ \\
\hline \multicolumn{8}{|c|}{ Group $\times$ condition interaction } \\
\hline Superior temporal gyrus & 22 & 88 & 46 & -38 & 4 & 4.10 & $p<0.001$ \\
\hline Inferior frontal gyrus & 46 & 62 & 58 & 34 & 8 & 3.77 & $p<0.001$ \\
\hline Inferior frontal gyrus & 47 & & 54 & 34 & -4 & 3.35 & 0.001 \\
\hline Superior temporal gyrus & 22 & 31 & 48 & -58 & 14 & 2.97 & 0.002 \\
\hline
\end{tabular}

ToM affective Theory of Mind, Emo emotion recognition, Neutral neutral face processing. Subcluster peaks are inserted as well as between age of onset and performance in affective ToM $(r=-.58, p=0.005)$, and emotion recognition $(r=-.54, p=0.009)$.

\section{Functional brain imaging results}

A main effect of task with increasing activation from neutral face processing over emotion recognition to affective ToM was found in regions of the "social brain", namely in clusters of the middle and superior temporal gyri, covering the pSTS region bilaterally, in the inferior prefrontal gyrus bilaterally and in the right amygdala (Table 3). In addition, a significant group by social-cognitive function interaction occurred in a cluster in right temporal gyrus, covering the pSTS region and right inferior prefrontal cortex (Table 3; Fig. 2) that was caused by schizophrenia patients having increased activation in the neutral face processing condition and by lacking an increase in activation from neutral face processing over emotion recognition to affective ToM that was evident in the healthy control group. This cluster of the group by social-cognitive function interaction, covering the right pSTS, was used for further post hoc tests and as seed region for the connectivity analyses. Post hoc $t$ tests with the first eigenvariate of right pSTS activation revealed a significant hyperactivation during neutral face processing $[t(d f 42)=3.18, p=0.003, d=.94]$, as well as a significant hypoactivation for emotion recognition $[t$ $(d f 42)=-2.28, p=0.028, d=.71]$ and affective ToM $[t$ $(d f 42)=2.46, p=0.017,-d=.75]$ in the schizophrenia group in comparison with the healthy control group. There neither was a significant correlation between pSTS activation and medication, as assessed by chlorpromazine equivalents, nor between pSTS activation and psychopathology, or duration of illness or age of onset. In the patient group, there was an association between activity during neutral face processing and reaction times for neutral face processing $(r=-.439, p=0.041)$, and emotion recognition $(r=-.502, p=0.017)$. In the healthy control group, the MSCEIT emotions scale was associated with activity during neutral face processing $(r=.498, p=0.018)$. However, none of these correlations survived correction for multiple testing.

The gPPI-analysis showed a significant interaction of group and social-cognitive function, too. In comparison with the healthy control group, patients with schizophrenia showed less increase in connectivity from neutral face processing 
(a)

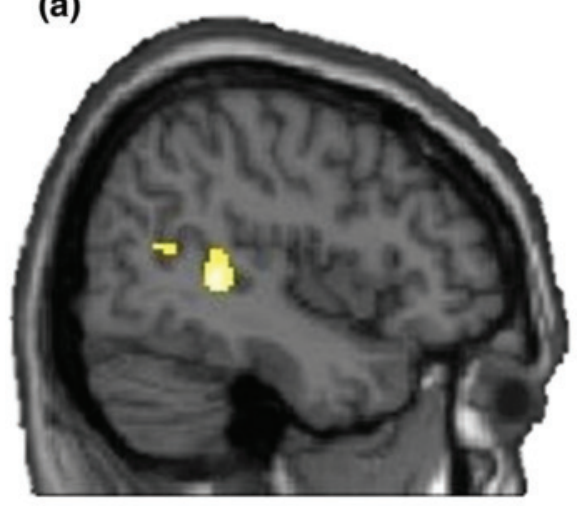

(b)

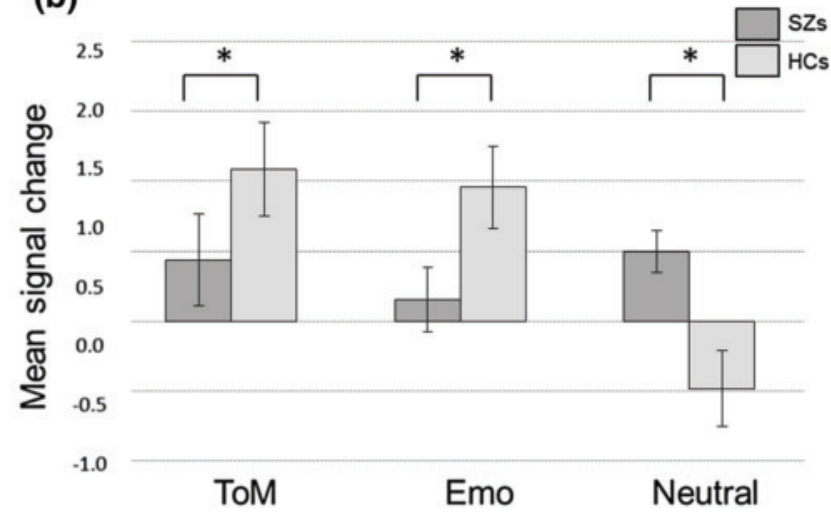

Fig. 2 Significant social-cognitive function by group interaction in activity. a Display of activity differences in right posterior superior temporal sulcus ( $p<0.005$, uncorrected) and b mean signal change (with respect to the fixation cross baseline) within the right posterior superior temporal sulcus

(a)

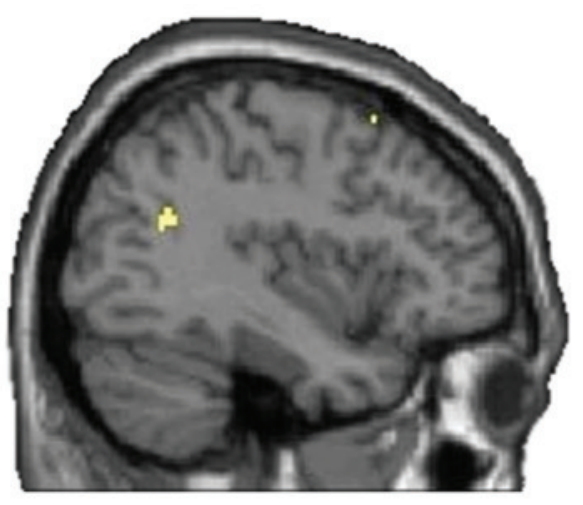

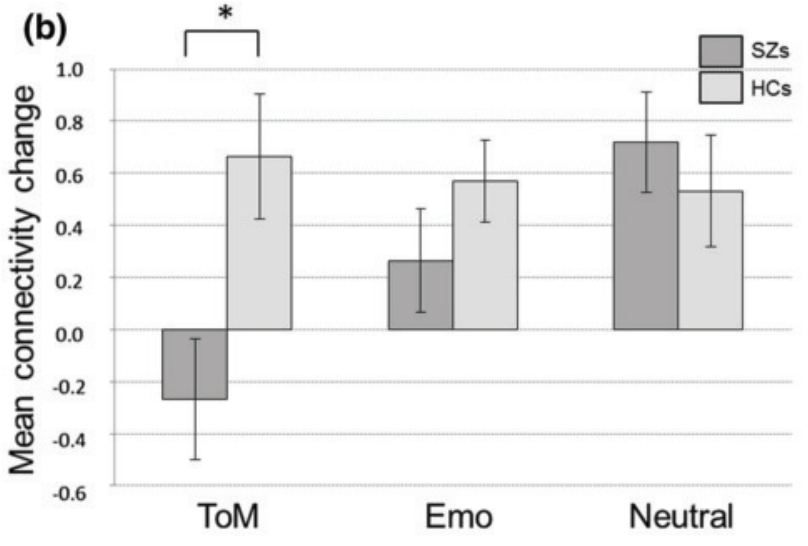

(c)
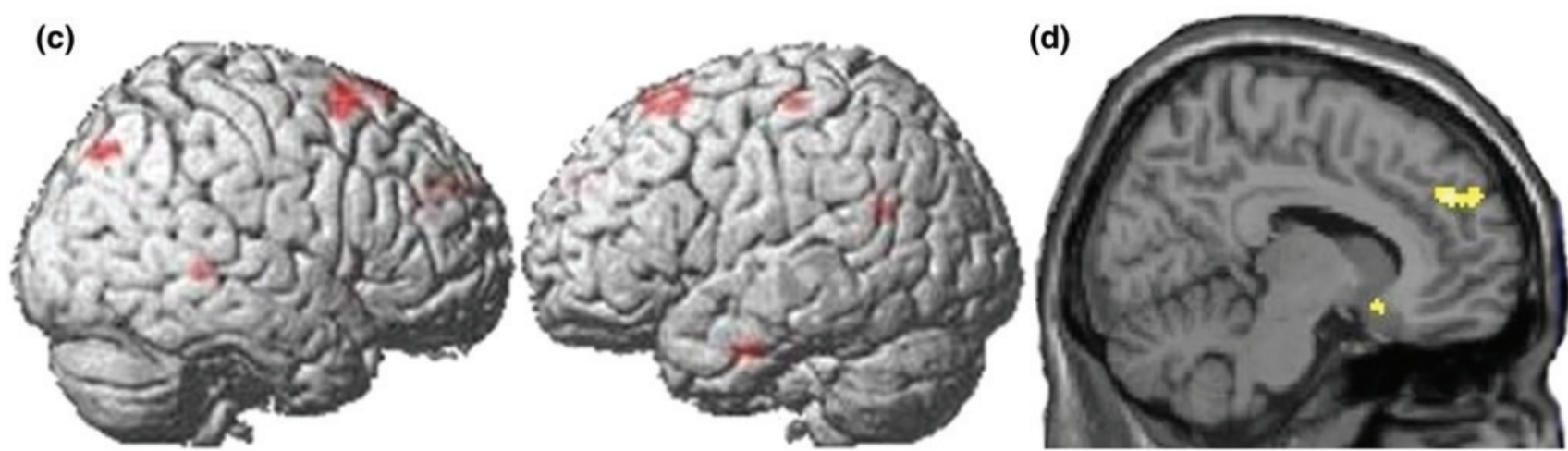

Fig. 3 Significant social-cognitive function by group interaction in connectivity of the right posterior superior temporal sulcus. a Display of connectivity differences from right to left posterior superior temporal sulcus ( $p<0.005$, uncorrected), b mean signal change (with respect to the fixation cross baseline) of connectivity of right to left

over emotion recognition to affective ToM between right and left pSTS (Fig. 3), as well as between right pSTS and right medial prefrontal cortex, premotor cortex bilaterally, visual cortex, putamen and somatosensory cortex (Table 4). Post hoc posterior superior temporal sulcus, $\mathbf{c}$ display of the social-cognitive function by group interaction in cortical brain regions $(p<0.005$, uncorrected) and $\mathbf{d}$ display of the social-cognitive function by group interaction in the medial frontal cortex $(p<0.005$, uncorrected)

$t$ tests were performed with the first eigenvariate of left pSTS, showing a significant hypoconnectivity for the patient group for affective ToM $[t(d f 42)=2.76, p=0.009, d=.83]$, but not for emotion recognition or neutral faces. There was 
Table 4 Group by condition interaction, as revealed by the generalized psychophysiological interactions $(p<0.005$ uncorrected, $k=20$ )

\begin{tabular}{|c|c|c|c|c|c|c|c|}
\hline \multirow{2}{*}{$\begin{array}{l}\text { Group } \times \text { condition interaction } \\
\text { Area }\end{array}$} & \multirow[t]{2}{*}{$\mathrm{BA}$} & \multirow[t]{2}{*}{ Cluster } & \multicolumn{3}{|l|}{ MNI } & \multirow[t]{2}{*}{$t$ value } & \multirow[t]{2}{*}{$p$ value } \\
\hline & & & $x$ & $y$ & $z$ & & \\
\hline Middle temporal gyrus & 22 & 40 & 52 & -40 & 0 & 3.87 & $p<0.001$ \\
\hline Middle temporal gyrus & 21 & & 62 & -38 & 0 & 3.01 & 0.002 \\
\hline Medial frontal gyrus & 9 & 79 & 10 & 46 & 32 & 3.75 & $p<0.001$ \\
\hline Medial frontal gyrus & 9 & & 16 & 46 & 22 & 3.20 & 0.001 \\
\hline Anterior cingulate & 25 & 31 & 6 & 14 & -10 & 3.59 & $p<0.001$ \\
\hline Superior frontal gyrus & 6 & 113 & -10 & 30 & 64 & 3.51 & $p<0.001$ \\
\hline Superior frontal gyrus & 6 & & -6 & 22 & 68 & 3.30 & 0.001 \\
\hline Middle frontal gyrus & 6 & & -34 & 18 & 60 & 3.18 & 0.001 \\
\hline Superior temporal gyrus & 39 & 47 & -38 & -56 & 24 & 3.44 & $p<0.001$ \\
\hline Postcentral gyrus & 3 & 23 & -44 & -26 & 62 & 3.43 & $p<0.001$ \\
\hline Precuneus & 19 & 35 & 44 & -74 & 44 & 3.34 & 0.001 \\
\hline Superior frontal gyrus & 6 & 88 & 26 & 12 & 68 & 3.31 & 0.001 \\
\hline Middle frontal gyrus & 6 & & 40 & 10 & 60 & 3.22 & 0.001 \\
\hline Middle frontal gyrus & 6 & & 32 & 18 & 62 & 3.03 & 0.002 \\
\hline Fusiform gyrus & 20 & 25 & -60 & -6 & -28 & 3.24 & 0.001 \\
\hline Putamen & & 25 & 24 & 10 & 12 & 3.13 & 0.001 \\
\hline
\end{tabular}

Subcluster peaks are inserted a significant negative correlation between pSTS-pSTS connectivity during affective ToM and medication $(r=-.442$, $p=0.045$ ), as assessed by chlorpromazine equivalents. There were several significant associations between performance and pSTS-pSTS connectivity in the patient group: pSTSpSTS connectivity for neutral face processing was associated with performance in affective ToM $(r=.436, p=0.043)$. In addition, emotion recognition performance correlated with pSTS-pSTS connectivity in all three social-cognitive functions (affective ToM: $r=.448, p=0.036$, emotion recognition: $r=.497, p=0.019$, neutral face processing: $r=.565$, $p=0.006)$. PANSS positive pathology was linked to pSTSpSTS connectivity during emotion recognition in the patient group $(r=.512, p=0.015)$. There were no significant associations between pSTS-pSTS connectivity and duration of illness, or age of onset. In the healthy control group, selfreported empathy was associated with pSTS-pSTS connectivity during affective ToM $(r=.473, p=0.026)$. Reaction times for affective ToM in the healthy control group were correlated with pSTS activity during emotion recognition $(r=-.529, p=0.011)$. However, none of these correlations survived correction for multiple testing.

Resting-state connectivity analyses showed a significantly reduced connectivity of the right pSTS to the postcentral gyrus bilaterally, the cerebellum, the thalamus, as well as the left inferior frontal gyrus in the schizophrenia group in comparison with the healthy control group (Table 5). However, no altered connectivity from right pSTS to left pSTS was evident.
Results from additional analyses (affective ToM $>$ emotion recognition, affective ToM $>$ neutral face processing, emotion recognition $>$ neutral face processing; affective ToM > emotion recognition $>$ neutral face processing separately for the groups; group comparisons for affective ToM, emotion recognition and neutral face processing; differences between groups for right pSTS to left pSTS connectivity depending on task vs. rest) are given in the supplementary materials (supplementary figures 1 and 2, supplementary tables 1-10 and supplementary text).

\section{Discussion}

The present investigation aimed at examining pSTS activity and connectivity in schizophrenia. We applied a social-cognitive task and a resting-state measurement to a group of patients with schizophrenia, as well as to a matched healthy control group. We replicated previous findings of aberrant pSTS activation in schizophrenia during social cognition and extended these findings by showing aberrant connectivity changes of the right to the left pSTS in task conditions, but not during rest.

First of all, we replicated previous findings of deficient social cognition with our current schizophrenia sample $[4,6,58]$. Regarding the number of correct responses, the patient group performed significantly inferior to the healthy control group in the fMRI task, investigating emotion recognition and affective ToM. Patients, however, showed 
Table 5 Reduced connectivity of the right posterior superior temporal sulcus in schizophrenia patients under rest $(p<0.005$ uncorrected, $k=20)$

\begin{tabular}{|c|c|c|c|c|c|c|c|}
\hline \multirow{2}{*}{$\begin{array}{l}H C>S Z \\
\text { Area }\end{array}$} & \multirow[t]{2}{*}{ BA } & \multirow[t]{2}{*}{ Cluster } & \multicolumn{3}{|l|}{ MNI } & \multirow[t]{2}{*}{$t$ value } & \multirow[t]{2}{*}{$p$ value } \\
\hline & & & $x$ & $y$ & $z$ & & \\
\hline Thalamus & & 45 & 6 & -22 & 7 & 4.04 & $p<0.001$ \\
\hline Postcentral gyrus & 3 & 89 & 66 & -19 & 28 & 3.90 & $p<0.001$ \\
\hline Postcentral gyrus & 2 & & 48 & -28 & 37 & 2.94 & 0.003 \\
\hline Cerebellum & & 36 & -27 & -73 & -50 & 3.82 & $p<0.001$ \\
\hline Cerebellum & & & -18 & -76 & -38 & 3.04 & 0.002 \\
\hline Inferior frontal gyrus & 46 & 27 & -36 & 41 & 7 & 3.58 & 0.001 \\
\hline Postcentral gyrus & 40 & 63 & -33 & -37 & 55 & 3.47 & 0.001 \\
\hline Postcentral gyrus & 2 & & -33 & -31 & 40 & 3.26 & 0.001 \\
\hline Cerebellum & & 38 & 9 & -22 & -38 & 3.26 & 0.001 \\
\hline Cerebellum & & & 12 & -37 & -41 & 3.24 & 0.001 \\
\hline Cerebellum & & & 18 & -40 & -50 & 3.08 & 0.002 \\
\hline
\end{tabular}

The reverse contrast revealed no significance under the given significance threshold. Subcluster peaks are inserted

$S Z$ patients with schizophrenia, $H C$ healthy control subjects no significant aberrations in self-reported empathy, in the RMET, as well as in the MSCEIT.

In the fMRI data, a group by social-cognitive function interaction occurred with patients in comparison with the healthy controls showing hypoactivation of right pSTS and right inferior prefrontal gyrus during affective ToM and emotion recognition, and hyperactivation for neutral face processing, partially replicating our previous finding from a group of schizophrenia outpatients [4], as well as findings from other groups indicating pSTS hypoactivity in response to emotionally relevant social stimuli, but not for emotionally irrelevant ones [23]. Moreover, we found decreased connectivity between right and left pSTS for affective ToM, but not for emotion recognition and neutral face processing in the schizophrenia group. The pSTS/temporo-parietal junction is directly involved in mental-state reasoning [59, 60] and is one of the main structures consistently reported in fMRI studies investigating ToM [61]. Analysis of the contrast estimates shows that the aberrant connectivity of the pSTS was due to a linear decrease of connectivity from neutral face processing to affective ToM in the schizophrenia group, but an increase in the healthy control group. A comparable pattern was found in the study by Straube and colleagues $(2013,2014)$. The authors reported reduced activity for the processing of metaphoric gestures in left pSTS and left inferior prefrontal gyrus in schizophrenia patients [62]. In a follow-up analysis, the authors found that this pattern of activation went along with reduced connectivity of the left pSTS with the inferior frontal gyrus [31]. These results could be explained by enhanced activity, respectively, and connectivity, of the left pSTS for iconic gestures. In agreement with the findings of Straube and colleagues, Ciaramidaro found increased pSTS activity, as well as increased pSTS connectivity in the control condition of a ToM-task [30]. Our findings, together with the findings of Straube and colleagues as well as those of Ciaramidaro and colleagues, could have at least two major implications. First of all, albeit there were only few significant correlations between activation and performance in our current sample, the reduced activity and reduced connectivity in the conditions that require enhanced activity/connectivity in areas of the social-cognitive network might be associated with deficits (i.e., patients perform worse in emotion recognition and affective ToM and show reduced activity in amygdala and pSTS, as well as reduced pSTS-pSTS connectivity). Second, the hyperactivity in the pSTS region in the tasks that do not need enhanced activity might make patients prone to false positives, i.e., the faulty perception of emotions and intentions. This pattern of false positives in social-cognitive tasks does not seem to be a new finding in the schizophrenia literature. However, it gets more attention since fMRI reveals congruent results. In 2003, Blakemore and colleagues already showed that patients with schizophrenia do not differentiate in their ratings of intentionality between animate random sequences and animate interactive sequences [63]. In line with this finding, Frith proposed that patients with schizophrenia may suffer from hypo- or hyper-ToM, depending on current psychopathology [64]. Hence, the hypoactivity, as well as hypoconnectivity might be causal to hypo-ToM, while the hyperactivity might result in hyper-ToM. In her review from 2012, Wible suggests an association between an overactive pSTS/temporo-parietal junction region and schizophrenia. In accordance, especially hyperactivity in response to neutral facial expressions can be assumed to facilitate the emergence of delusions. While we found some correlations in our data that might support this assumption, none of the correlations survived correction for multiple 
testing. Hence, future studies with larger samples sizes are warranted to investigate the link between social-cognitive performance and pSTS activity and connectivity.

To follow up on the assumption that aberrations in connectivity of the pSTS might reflect an intrinsic network property of the schizophrenia brain, we additionally analyzed resting-state connectivity of the right pSTS. Here, in comparison with the connectivity analysis for the socialcognitive task, we found no evidence for aberrant interhemispheric pSTS connectivity in schizophrenia. However, we found reduced connectivity to several other brain regions, including the MPFC that is thought to be the core region of the mentalizing network [65]. Importantly, this finding is in agreement with two recent studies by Schilbach and colleagues, showing reduced connectivity during rest in schizophrenia $[66,67]$. In these studies, the authors report reduced connectivity within the default mode network, as well as within the mentalizing and the mirror neuron system in schizophrenia. These results suggest that reduced connectivity of the pSTS is the default state in schizophrenia and does not occur while processing neutral facial expressions (and maybe social stimuli without an emotional or intentional significance in general). Hence, there is clear evidence for a relatively enhanced engagement of social brain structures for neutral facial expressions in schizophrenia [4, 23], but not during rest [66, 67]. In particular, the enhanced activity of the pSTS in response to the neutral facial expressions could result in the faulty perception of intentions (i.e., hypermentalizing), as has been suggested earlier [36].

The present study has several limitations. Our sample for the resting-state analyses was smaller than for the social-cognitive task, resulting in lower reliability than for the task-dependent connectivity analysis. Thus, these results have to be considered as preliminary. The smaller sample might also result in reduced power to find comparable aberrations in connectivity as detected in the taskdependent connectivity analysis. This, however, seems not to be the case, because the interaction pattern of socialcognitive function and group could also be revealed when only including the participants from the resting-state analysis. Moreover, connectivity differenced significantly in this sample between groups for affective ToM, but not for rest (see supplementary text). In addition, newer approaches to the analysis of the resting-state data that provide better control of movement influences (e.g., [68]) should be applied in future studies.

In the last years, the finding of amygdala hyperactivation in response to neutral facial expressions in schizophrenia has gathered growing attention [19]. It was assumed that this hyperactivation results in a negative bias in facial emotion perception [18]. However, we could not replicate our earlier finding of enhanced amygdala activation for emotion recognition and neutral facial expressions in schizophrenia, as well as for pSTS hyperactivation for emotion recognition [4]. In our earlier study, all patients were outpatients and were remitted from positive symptoms. The patients in the present study had higher variance in psychopathology, possibly reducing the power to detect more subtle group differences. In addition, although all patients were on a antipsychotic monotherapy, medication, as well as recent changes in dosage of medication, might have superimposed group differences. Importantly, while our finding of aberrant pSTS activity adds to studies suggesting social-cognitive deficits and alterations in pSTS functioning as an intermediate phenotype for schizophrenia, our lack of replication of aberrant amygdala activity, however, supports the finding that aberrations in amygdala activation are dependent on the state of disease, or psychopathology, cannot be observed during remission and therefore do not represent an intermediate phenotype of schizophrenia [24]. Hence, an investigation of drug-naïve, first-episode patients with a more homogeneous clinical pattern seems warranted. Moreover, acquiring larger patient samples that allow a comparison of subgroups, depending on their psychopathology, would help shedding light on the impact of amygdala functioning on social-cognitive deficits in schizophrenia.

Interestingly, we neither found a significant deficit in self-reported empathy in our schizophrenia patients, nor in the RMET, or in the MSCEIT. Performance in the MSCEIT has been shown to be linked to age of onset [69], as well as to cognitive abilities [70]. This link between performance in the MSCEIT and age of onset was revealed in our schizophrenia sample, too. Moreover, there is evidence that the RMET taps a social-cognitive ability in schizophrenia that is less severely impaired [71, 72], and recent studies indicate that patients with schizophrenia are only impaired in specific, but not all aspects of empathy [73]. These findings again add to the necessity to collect larger patient samples in future studies and to take current state of psychopathology into account to disentangle the social-cognitive deficit in schizophrenia. In addition, larger sample sizes would be important to address gender effects on social-cognitive abilities in schizophrenia.

Finally, it has to be kept in mind that our reported statistics are not corrected for multiple comparisons and that the selected significance threshold was chosen to be sensitive for group differences, but might have the risk of typeI errors, suggesting the need for replication (especially of the connectivity and correlational findings). In addition, it would be important for future studies to investigate social cognition across disorders. There is plenty of evidence that not only patients with schizophrenia, but also patients with other mental disorders, such as autism, or social phobia show aberrations in social cognition [15] and social 
interaction [74]. Hence, it can be assumed that deficits in social cognition present a transdiagnostic marker [15, 74]. Direct comparisons of different mental disorders are needed to disentangle common and distinct deficits.

The present study gives further evidence for a central role of the pSTS for the social-cognitive deficit in schizophrenia, supporting the assumption of altered pSTS functionality during social cognition as an intermediate phenotype for schizophrenia. We could show enhanced right pSTS activation during neutral face processing and reduced right $\mathrm{pSTS}$ activation during emotion recognition and affective ToM. Moreover, our data suggest that aberrant right to left pSTS connectivity in schizophrenia does not merely reflect an intrinsic network property in schizophrenia, but specifically occurs during task. In agreement with earlier studies, it can be assumed that an enhanced engagement of the pSTS in response to social stimuli without an affective meaning makes patients prone to hypermentalizing and in turn might present a vulnerability factor for the emergence of delusions. Future studies with drug-naïve patients with an at-risk-mental state for psychosis or during first manifestation can add to our understanding of the role of social cognition in the development of psychosis.

Acknowledgments We thank Dagmar Gass for her help with data collection. We are grateful to all participants and the staff of the Central Institute of Mental Health that assisted patient recruitment. The work was funded by the Deutsche Forschungsgemeinschaft (ZI1253/3-1, ZI1253/3-2, KI 576/14-2, ME 1591/6-2).

\section{Compliance with ethical standards}

Conflict of interest The authors declare that they have no conflict of interest.

\section{References}

1. Green MF, Bearden CE, Cannon TD, Fiske AP, Hellemann GS, Horan WP, Kee K, Kern RS, Lee J, Sergi MJ, Subotnik KL, Sugar CA, Ventura J, Yee CM, Nuechterlein KH (2012) Social cognition in schizophrenia, part 1: performance across phase of illness. Schizophr Bull 38(4):854-864. doi:10.1093/schbul/sbq171

2. Horan WP, Green MF, DeGroot M, Fiske A, Hellemann G, Kee K, Kern RS, Lee J, Sergi MJ, Subotnik KL, Sugar CA, Ventura J, Nuechterlein KH (2012) Social cognition in schizophrenia, part 2: 12-month stability and prediction of functional outcome in first-episode patients. Schizophr Bull 38(4):865-872. doi:10.1093/schbul/sbr001

3. Brothers L (1990) The social brain: a project for integrating primate behavior and neurophysiology in a new domain. Concepts Neurosci 1(1):27-51

4. Mier D, Sauer C, Lis S, Esslinger C, Wilhelm J, Gallhofer B, Kirsch P (2010) Neuronal correlates of affective theory of mind in schizophrenia out-patients: evidence for a baseline deficit. Psychol Med 40(10):1607-1617. doi:10.1017/ S0033291709992133
5. Turetsky BI, Kohler CG, Indersmitten T, Bhati MT, Charbonnier D, Gur RC (2007) Facial emotion recognition in schizophrenia: when and why does it go awry? Schizophr Res 94(1-3):253-263. doi:10.1016/j.schres.2007.05.001

6. Edwards J, Jackson HJ, Pattison PE (2002) Emotion recognition via facial expression and affective prosody in schizophrenia: a methodological review. Clin Psychol Rev J 22(6):789-832

7. Brune M (2005) Emotion recognition, 'theory of mind', and social behavior in schizophrenia. Psychiatry Res 133(2-3):135147. doi:10.1016/j.psychres.2004.10.007

8. Premack D, Woodruff G (1978) Does the chimpanzee have a theory of mind? Behav Brain Sci 4:515-526

9. Shamay-Tsoory SG, Shur S, Barcai-Goodman L, Medlovich S, Harari H, Levkovitz Y (2007) Dissociation of cognitive from affective components of theory of mind in schizophrenia. Psychiatry Res 149(1):11-23

10. Mehta UM, Thirthalli J, Naveen Kumar C, Keshav Kumar J, Keshavan MS, Gangadhar BN (2013) Schizophrenia patients experience substantial social cognition deficits across multiple domains in remission. Asian J Psychiatry 6(4):324-329. doi:10.1016/j.ajp.2013.02.001

11. Amminger GP, Schafer MR, Papageorgiou K, Klier CM, Schlogelhofer M, Mossaheb N, Werneck-Rohrer S, Nelson B, McGorry PD (2012) Emotion recognition in individuals at clinical high-risk for schizophrenia. Schizophr Bull 38(5):10301039. doi: $10.1093 /$ schbul/sbr015

12. Eack SM, Mermon DE, Montrose DM, Miewald J, Gur RE, Gur RC, Sweeney JA, Keshavan MS (2010) Social cognition deficits among individuals at familial high risk for schizophrenia. Schizophr Bull 36(6):1081-1088. doi:10.1093/schbul/sbp026

13. Meyer-Lindenberg A (2010) Intermediate or brainless phenotypes for psychiatric research? Psychol Med 40(7):1057-1062

14. Meyer-Lindenberg A, Weinberger DR (2006) Intermediate phenotypes and genetic mechanisms of psychiatric disorders. Nat Rev Neurosci 7(10):818-827. doi:10.1038/nrn1993

15. Derntl B, Habel U (2011) Deficits in social cognition: a marker for psychiatric disorders? Eur Arch Psychiatry Clin Neurosci 261(Suppl 2):S145-S149. doi:10.1007/s00406-011-0244-0

16. Li H, Chan RC, McAlonan GM, Gong QY (2010) Facial emotion processing in schizophrenia: a meta-analysis of functional neuroimaging data. Schizophr Bull 36(5):1029-1039. doi: $10.1093 / \mathrm{schbul} / \mathrm{sbn} 190$

17. Hall J, Whalley HC, McKirdy JW, Romaniuk L, McGonigle D, McIntosh AM, Baig BJ, Gountouna VE, Job DE, Donaldson DI, Sprengelmeyer R, Young AW, Johnstone EC, Lawrie SM (2008) Overactivation of fear systems to neutral faces in schizophrenia. Biol Psychiatry 64(1):70-73. doi:10.1016/j. biopsych.2007.12.014

18. Mier D, Lis S, Zygrodnik K, Sauer C, Ulferts J, Gallhofer B, Kirsch P (2014) Evidence for altered amygdala activation in schizophrenia in an adaptive emotion recognition task. Psychiatry Res 221(3):195-203. doi:10.1016/j.pscychresns.2013.12.001

19. Anticevic A, Van Snellenberg JX, Cohen RE, Repovs G, Dowd EC, Barch DM (2012) Amygdala recruitment in schizophrenia in response to aversive emotional material: a meta-analysis of neuroimaging studies. Schizophr Bull 38(3):608-621. doi:10.1093/ schbul/sbq131

20. Hooker CI, Bruce L, Fisher M, Verosky SC, Miyakawa A, D'Esposito M, Vinogradov S (2013) The influence of combined cognitive plus social-cognitive training on amygdala response during face emotion recognition in schizophrenia. Psychiatry Res 213(2):99-107. doi:10.1016/j.pscychresns.2013.04.001

21. Hein G, Knight RT (2008) Superior temporal sulcus-it's my area: or is it? J Cogn Neurosci 20(12):2125-2136. doi:10.1162/ jocn.2008.20148 
22. Backasch B, Straube B, Pyka M, Klohn-Saghatolislam F, Muller MJ, Kircher TT, Leube DT (2013) Hyperintentionality during automatic perception of naturalistic cooperative behavior in patients with schizophrenia. Soc Neurosci 8(5):489-504. doi:10. 1080/17470919.2013.820666

23. Pinkham AE, Hopfinger JB, Pelphrey KA, Piven J, Penn DL (2008) Neural bases for impaired social cognition in schizophrenia and autism spectrum disorders. Schizophr Res 99(1-3):164175. doi:10.1016/j.schres.2007.10.024

24. Rasetti R, Mattay VS, Wiedholz LM, Kolachana BS, Hariri AR, Callicott JH, Meyer-Lindenberg A, Weinberger DR (2009) Evidence that altered amygdala activity in schizophrenia is related to clinical state and not genetic risk. Am J Psychiatry 166(2):216225. doi:10.1176/appi.ajp.2008.08020261

25. Walter H, Schnell K, Erk S, Arnold C, Kirsch P, Esslinger C, Mier D, Schmitgen MM, Rietschel M, Witt SH, Nothen MM, Cichon S, Meyer-Lindenberg A (2011) Effects of a genomewide supported psychosis risk variant on neural activation during a theory-of-mind task. Mol Psychiatry 16(4):462-470. doi: $10.1038 / \mathrm{mp} .2010 .18$

26. Wible CG (2012) Hippocampal temporal-parietal junction interaction in the production of psychotic symptoms: a framework for understanding the schizophrenic syndrome. Front Hum Neurosci 6:180. doi:10.3389/fnhum.2012.00180

27. Seeley WW, Menon V, Schatzberg AF, Keller J, Glover GH, Kenna H, Reiss AL, Greicius MD (2007) Dissociable intrinsic connectivity networks for salience processing and executive control. J Neurosci 27(9):2349-2356. doi:10.1523/ JNEUROSCI.5587-06.2007

28. Weiland BJ, Sabbineni A, Calhoun VD, Welsh RC, Hutchison KE (2014) Reduced executive and default network functional connectivity in cigarette smokers. Hum Brain Mapp. doi:10.1002/hbm.22672

29. Crossley NA, Mechelli A, Fusar-Poli P, Broome MR, Matthiasson P, Johns LC, Bramon E, Valmaggia L, Williams SC, McGuire PK (2009) Superior temporal lobe dysfunction and frontotemporal dysconnectivity in subjects at risk of psychosis and in first-episode psychosis. Hum Brain Mapp 30(12):41294137. doi:10.1002/hbm.20834

30. Ciaramidaro A, Bolte S, Schlitt S, Hainz D, Poustka F, Weber B, Bara BG, Freitag C, Walter H (2015) Schizophrenia and autism as contrasting minds: neural evidence for the hypohyper-intentionality hypothesis. Schizophr Bull 41(1):171-179. doi:10.1093/schbul/sbu124

31. Straube B, Green A, Sass K, Kircher T (2014) Superior temporal sulcus disconnectivity during processing of metaphoric gestures in schizophrenia. Schizophr Bull 40(4):936-944. doi:10.1093/ schbul/sbt110

32. Anticevic A, Repovs G, Barch DM (2012) Emotion effects on attention, amygdala activation, and functional connectivity in schizophrenia. Schizophr Bull 38(5):967-980. doi:10.1093/ schbul/sbq168

33. Mukherjee P, Whalley HC, McKirdy JW, Sprengelmeyer R, Young AW, McIntosh AM, Lawrie SM, Hall J (2014) Altered amygdala connectivity within the social brain in schizophrenia. Schizophr Bull 40(1):152-160. doi:10.1093/schbul/sbt086

34. Adolphs R (2009) The social brain: neural basis of social knowledge. Annu Rev Psychol 60:693-716. doi:10.1146/annurev. psych.60.110707.163514

35. Blakemore SJ (2008) The social brain in adolescence. Nat Rev Neurosci 9(4):267-277. doi:10.1038/nrn2353

36. Frith CD, Corcoran R (1996) Exploring 'theory of mind' in people with schizophrenia. Psychol Med 26(3):521-530

37. Lee SM, Gao T, McCarthy G (2014) Attributing intentions to random motion engages the posterior superior temporal sulcus. Soc Cogn Affect Neurosci 9(1):81-87. doi:10.1093/scan/nss110
38. Mier D, Lis S, Neuthe K, Sauer C, Esslinger C, Gallhofer B, Kirsch P (2010) The involvement of emotion recognition in affective theory of mind. Psychophysiology 47(6):1028-1039. doi:10.1111/j.1469-8986.2010.01031.x

39. Rausch F, Mier D, Eifler S, Esslinger C, Schilling C, Schirmbeck F, Englisch S, Meyer-Lindenberg A, Kirsch P, Zink M (2014) Reduced activation in ventral striatum and ventral tegmental area during probabilistic decision-making in schizophrenia. Schizophr Res 156(2-3):143-149. doi:10.1016/j.schres.2014.04.020

40. Rausch F, Mier D, Eifler S, Fenske S, Schirmbeck F, Englisch S, Schilling C, Meyer-Lindenberg A, Kirsch P, Zink M (2015) Reduced activation in the ventral striatum during probabilistic decision-making in patients in an at-risk mental state. J Psychiatry Neurosci JPN 40(3):163-173

41. American Psychiatric Association (2000) Diagnostic and statistical manual of mental disorders DSM-IV-TR fourth edition (text revision)

42. Kay SR, Fiszbein A, Opler LA (1987) The positive and negative syndrome scale (PANSS) for schizophrenia. Schizophr Bull 13(2):261-276

43. Andreasen NC (1989) The Scale for the Assessment of Negative Symptoms (SANS): conceptual and theoretical foundations. Br J Psychiatry (7):49-58

44. Patrick DL, Burns T, Morosini P, Rothman M, Gagnon DD, Wild D, Adriaenssen I (2009) Reliability, validity and ability to detect change of the clinician-rated Personal and Social Performance scale in patients with acute symptoms of schizophrenia. Curr Med Res Opin 25(2):325-338. doi:10.1185/03007990802611919

45. Sheehan DV, Lecrubier Y, Sheehan KH, Amorim P, Janavs J, Weiller E, Hergueta T, Baker R, Dunbar GC (1998) The MiniInternational Neuropsychiatric Interview (M.I.N.I.): the development and validation of a structured diagnostic psychiatric interview for DSM-IV and ICD-10. J Clin Psychiatry 59(Suppl 20):22-33 (quiz 34-57)

46. Lehrl S (2005) Mehrfachwahl-Wortschatz-Intelligenztest MWTB. Spitta Verlag, Bailingen, Germany

47. Kern RS, Gold JM, Dickinson D, Green MF, Nuechterlein KH, Baade LE, Keefe RS, Mesholam-Gately RI, Seidman LJ, Lee C, Sugar CA, Marder SR (2011) The MCCB impairment profile for schizophrenia outpatients: results from the MATRICS psychometric and standardization study. Schizophr Res 126(1-3):124131. doi:10.1016/j.schres.2010.11.008

48. Nuechterlein KH, Green MF, Kern RS, Baade LE, Barch DM, Cohen JD, Essock S, Fenton WS, Frese FJ 3rd, Gold JM, Goldberg T, Heaton RK, Keefe RS, Kraemer H, Mesholam-Gately R, Seidman LJ, Stover E, Weinberger DR, Young AS, Zalcman S, Marder SR (2008) The MATRICS Consensus Cognitive Battery, part 1: test selection, reliability, and validity. Am J Psychiatry 165(2):203-213. doi:10.1176/appi.ajp.2007.07010042

49. Baron-Cohen S, Jolliffe T, Mortimore C, Robertson M (1997) Another advanced test of theory of mind: evidence from very high functioning adults with autism or asperger syndrome. $\mathrm{J}$ Child Psychol Psychiatry 38(7):813-822

50. Tran US, Laireiter AR, Schmitt DP, Neuner C, Leibetseder M, Szente-Voracek SL, Voracek M (2013) Factorial structure and convergent and discriminant validity of the E (Empathy) scale. Psychol Rep 113(2):441-463. doi:10.2466/03.02. PR0.113x20z9

51. Balogh N, Egerhazi A, Berecz R, Csukly G (2014) Investigating the state-like and trait-like characters of social cognition in schizophrenia: a short term follow-up study. Schizophr Res 159(2-3):499-505. doi:10.1016/j.schres.2014.08.027

52. Csukly G, Polgar P, Tombor L, Benkovits J, Rethelyi J (2014) Theory of mind impairments in patients with deficit schizophrenia. Compr Psychiatry 55(2):349-356. doi:10.1016/j. comppsych.2013.08.025 
53. Mier D, Lis S, Esslinger C, Sauer C, Hagenhoff M, Ulferts J, Gallhofer B, Kirsch P (2013) Neuronal correlates of social cognition in borderline personality disorder. Soc Cogn Affect Neurosci 8(5):531-537. doi:10.1093/scan/nss028

54. McLaren DG, Ries ML, Xu G, Johnson SC (2012) A generalized form of context-dependent psychophysiological interactions (gPPI): a comparison to standard approaches. NeuroImage 61(4):1277-1286. doi:10.1016/j.neuroimage.2012.03.068

55. van Buuren M, Gladwin TE, Zandbelt BB, van den Heuvel M, Ramsey NF, Kahn RS, Vink M (2009) Cardiorespiratory effects on default-mode network activity as measured with fMRI. Hum Brain Mapp 30(9):3031-3042. doi:10.1002/hbm.20729

56. Lieberman MD, Cunningham WA (2009) Type I and Type II error concerns in fMRI research: re-balancing the scale. Soc Cogn Affect Neurosci 4(4):423-428. doi:10.1093/scan/nsp052

57. Woods SW (2003) Chlorpromazine equivalent doses for the newer atypical antipsychotics. J Clin Psychiatry 64(6):663-667

58. Pinkham AE (2014) Social cognition in schizophrenia. J Clin Psychiatry 75(Suppl 2):14-19. doi:10.4088/JCP.13065su1.04

59. Samson D, Apperly IA, Chiavarino C, Humphreys GW (2004) Left temporoparietal junction is necessary for representing someone else's belief. Nat Neurosci 7(5):499-500. doi:10.1038/ nn1223

60. Mahy CE, Moses LJ, Pfeifer JH (2014) How and where: theoryof-mind in the brain. Dev Cogn Neurosci 9:68-81. doi:10.1016/j. den.2014.01.002

61. Mar RA (2011) The neural bases of social cognition and story comprehension. Annu Rev Psychol 62:103-134. doi:10.1146/ annurev-psych-120709-145406

62. Straube B, Green A, Sass K, Kirner-Veselinovic A, Kircher T (2013) Neural integration of speech and gesture in schizophrenia: evidence for differential processing of metaphoric gestures. Hum Brain Mapp 34(7):1696-1712. doi:10.1002/hbm.22015

63. Blakemore SJ, Sarfati Y, Bazin N, Decety J (2003) The detection of intentional contingencies in simple animations in patients with delusions of persecution. Psychol Med 33(8):1433-1441

64. Frith CD (2004) Schizophrenia and theory of mind. Psychol Med 34(3):385-389

65. Amodio DM, Frith CD (2006) Meeting of minds: the medial frontal cortex and social cognition. Nat Rev Neurosci 7(4):268277. doi:10.1038/nrn1884
66. Schilbach L, Derntl B, Aleman A, Caspers S, Clos M, Diederen KM, Gruber O, Kogler L, Liemburg EJ, Sommer IE, Muller VI, Cieslik EC, Eickhoff SB (2016) Differential patterns of dysconnectivity in mirror neuron and mentalizing networks in schizophrenia. Schizophr Bull 42(5):1135-1148. doi:10.1093/schbul/ sbw015

67. Schilbach L, Hoffstaedter F, Muller V, Cieslik EC, GoyaMaldonado R, Trost S, Sorg C, Riedl V, Jardri R, Sommer I, Kogler L, Derntl B, Gruber O, Eickhoff SB (2016) Transdiagnostic commonalities and differences in resting state functional connectivity of the default mode network in schizophrenia and major depression. NeuroImage Clin 10:326-335. doi:10.1016/j. nicl.2015.11.021

68. Power JD, Mitra A, Laumann TO, Snyder AZ, Schlaggar BL, Petersen SE (2014) Methods to detect, characterize, and remove motion artifact in resting state fMRI. NeuroImage 84:320-341. doi:10.1016/j.neuroimage.2013.08.048

69. Linke M, Jankowski KS, Ciołkiewicz A, Jędrasik-Styła M, Parnowska D, Gruszka A, Denisiuk M, Jarema M, Wichniak A (2015) Age or age at onset? Which of them really matters for neuro and social cognition in schizophrenia? Psychiatry Res 225(1):197-201

70. Frajo-Apor B, Pardeller S, Kemmler G, Welte A-S, Hofer A (2016) Emotional Intelligence deficits in schizophrenia: the impact of non-social cognition. Schizophr Res 172(1):131-136

71. Scherzer P, Achim A, Léveillé E, Boisseau E, Stip E (2015) Evidence from paranoid schizophrenia for more than one component of theory of mind. Front Psychol 6:1643. doi:10.3389/ fpsyg.2015.01643

72. Scherzer PB, Leveillé E, Achim A, Boisseau E, Stip E (2012) A study of theory of mind in paranoid schizophrenia: a theory or many theories? Front Psychol 3:432. doi:10.3389/ fpsyg.2012.00432

73. Lehmann A, Bahcesular K, Brockmann EM, Biederbick SE, Dziobek I, Gallinat J, Montag C (2014) Subjective experience of emotions and emotional empathy in paranoid schizophrenia. Psychiatry Res 220(3):825-833. doi:10.1016/j. psychres.2014.09.009

74. Schilbach L (2016) Towards a second-person neuropsychiatry. Philos Trans R Soc Lond B Biol Sci 371(1686):20150081. doi:10.1098/rstb.2015.0081 